\title{
Anthrax Lethal Toxin Inhibits the Production of Proinflammatory Cytokines
}

\author{
Aiguo Wu, ${ }^{1}$ Joseph Shiloach, ${ }^{2}$ Darya Alibek, ${ }^{1}$ \\ Lydia Yue Li, ${ }^{1}$ Christopher Bradburne, ${ }^{3}$ and Ken Alibek ${ }^{1}$ \\ ${ }^{1}$ AFG Biosolutions Inc., 9119 Gaither Road, Gaithersburg, MD 20877, USA \\ ${ }^{2}$ Biotechnology Unit, NIDDK, NIH, Building 6 Room B1-33, Bethesda, MD 20892, USA \\ ${ }^{3}$ Applied Physics Laboratory, Johns Hopkins University, MSN 8-146, 11100 Johns Hopkins Road, Laurel, MD 20723, USA
}

Correspondence should be addressed to Aiguo Wu; wuag888@gmail.com

Received 11 January 2013; Revised 5 March 2013; Accepted 6 March 2013

Academic Editor: Andreimar M. Soares

Copyright (C) 2013 Aiguo Wu et al. This is an open access article distributed under the Creative Commons Attribution License, which permits unrestricted use, distribution, and reproduction in any medium, provided the original work is properly cited.

\begin{abstract}
In previous studies, we have found that anthrax lethal toxin (LeTx) induces apoptosis in both murine macrophages and human peripheral blood mononuclear cells (PBMCs). In this study, we further report that bacterial cell wall (CW) components of Bacillus (B.) anthracis are powerful inducers of proinflammatory cytokines from the PBMCs. These effects are deprived when the LeTx is present. The major causative element for this suppression is lethal factor (LF) rather than protective antigen (PA). These results indicate that the roles of LeTx in anthrax pathogenesis, particularly its effects on cytokine production, should be reevaluated as our findings and other reports are controversial to the conventional concept.
\end{abstract}

\section{Introduction}

Anthrax is initiated by infection with B. anthracis, a Grampositive, spore forming bacterium. Infection occurs through three primary routes: cutaneous, ingestion, and inhalation of the spores. Systemic infection by inhalation of the spores has become a major research focus due to several reasons. First and foremost is the likelihood that B. anthracis spores would be used as a biological weapon due to its ease of growth, long shelf life, stability in the environment, and the ability to be effectively disseminated in a large area to inflict high mortality. Furthermore, the inhalation form can cause septic shock that has a mortality rate approaching $100 \%$ with death occurring within a few days after onset of symptoms if vigorous treatment is not given immediately after exposure. Lastly, to date, there is no effective treatment for the latestage infection and it is usually fatal, even with aggressive antibiotic therapy [1]. Therefore, given these factors, in conjunction with the increased threat of a biological attack, it is imperative that the true pathogenesis of this microbe should be understood so that new successful treatment methods can be developed.
The virulence of $B$. anthracis depends on three plasmidencoded factors: two secreted protein toxins and an antiphagocytic poly-D-glutamic acid capsule. The two toxins, collectively called anthrax toxin, are formed by the binary association of three proteins: PA $(83 \mathrm{kDa}), \mathrm{LF}$ $(90 \mathrm{kDa})$, and edema factor $(\mathrm{EF}, 89 \mathrm{kDa})$. PA either combines with LF to form lethal toxin (LeTx) or with EF to form edema toxin (EdTx) [2-4]. LeTx is considered to be responsible for the lysis of macrophages, which is accompanied by the release of cytokines, specifically tumor-necrosis-factor (TNF-) $\alpha$ and interleukin- (IL-) $1 \beta$. These cytokines are considered to be the major players responsible for septic shock, and sudden death in systemic anthrax infection [5-7]. EdTx toxin is thought to promote bacterial survival during early stages of anthrax infection since it inhibits neutrophil function in vitro by inhibiting phagocytosis of opsonized, avirulent $B$. anthracis spores [8]. EdTx is therefore acting synergistically with LeTx to promote the progression of the infection to perpetuate a fatal outcome.

The current view of anthrax pathogenesis suggests that LeTx is primarily responsible for the massive shock and 
death associated with systemic anthrax infection. There is no doubt that these toxins are essential in anthrax infection. However, they may be less critical in the resultant sepsis, shock and death than once understood. PA is regarded as the principle protective element in natural or vaccine-induced immunity; though when administered alone, it induces a poor humoral response. Furthermore, a serum anti-PA level cannot be used to predict whether there is true protection [9]. In addition, the capability of LeTx to stimulate cytokine production is not certain [10-12]. These findings together with the observations that LeTx is highly unstable in vivo and lacks the capability to lyse many monocyte-macrophage cell lines suggest that other virulence factors, in addition to LeTx, may play important roles during the course of anthrax infection. The anthrax CW, which to date has not been studied extensively, could be one of those virulence factors. The concept of bacterial cell walls playing a role in the pathogenesis of bacterial infections is not new. It is well known that the lipopolysaccharide (LPS), constituent of Gram-negative bacteria cell walls, promotes sepsis and shock by inducing endogenous shock mediators [13]. Numerous proinflammatory cytokine-inducing molecules are present in Gram-positive bacteria such as staphylococcus, streptococcus, and mycobacterium [14]. The modulins found in Grampositive organisms including Teichoic, Lipoteichoic Acids, and peptidoglycan stimulate the production of IL- $1 \beta$, TNF$\alpha$, IL-6, and granulocyte colony-stimulating factor $[15,16]$.

The objective of this work was to study the effects of the CW components on the production of the proinflammatory cytokines in human PBMCs and to compare the effects with those of LeTx. In addition, the interactive effects of CW components and LeTx on inducing production of the proinflammatory cytokines were evaluated. We show here that the causative agents for the cytokine production are from the bacterial CW rather than LeTx. Furthermore, the production of the proinflammatory cytokine induced by bacterial CW can be significantly inhibited in the presence of LeTx. When compared to studies on individual cell types in the literature, our results indicate that complex populations of human immune cells, such as PBMCs, may present an entirely different phenotype, and that this phenotype is more relevant to overall systemic anthrax disease. This study reveals that B. anthracis CW has a role in anthrax pathogenesis and may provide new clues in searching novel treatment and proper candidates for anthrax vaccine development.

\section{Materials and Methods}

2.1. Human Cells Preparation. Peripheral blood was collected from normal volunteer donors. The Department of Health and Human Services/NIH guidelines on protection of human subjects were followed with the authorization of the George Mason University Institutional Review Board. The blood was drawn into a $50 \mathrm{~mL}$ conical tube containing EDTA $(1.8 \mathrm{mg} / \mathrm{mL})$. Ten milliliter of blood was diluted with $20 \mathrm{~mL}$ of phosphate buffered saline (PBS) and layered on Ficoll/LiteLymphoH (1.077, Atlanta Biologicals, Atlanta, GA, USA). The PBMCs were separated by a density centrifugation at $900 \mathrm{~g}$ for $30 \mathrm{~min}$ on Ficoll/Lite. The buffy coat was collected and washed twice with RPMI-1640 (Invitrogen, Carlsbad, CA, USA) containing 10\% Fetal Bovine Serum (FBS) (Atlanta Biologicals). The PBMCs were resuspended to $10^{6}$ cells $/ \mathrm{mL}$ with phenol free RPMI-1640 medium.

2.2. Bacterial CW Preparation and Anthrax Toxin. An avirulent B. anthracis strain SNKE-FF-E308D was used for CW preparation. After growth, the CW was prepared according to Medvedev's protocol [17]. The amount of CW ranged between 30 and $35 \%$ of the dry weight. The CW preparations were checked for aerobic and anaerobic bacteria, endotoxin, and LPS contamination. Bacterial contamination was checked using various growth media. The endotoxin and LPS were tested using a Limulus amoebocyte lysate test kit (E-TOXATE, Sigma, St. Louis, MO, USA). No endotoxin and LPS were found in the preparations. PA, LF, and EF were purchased from List-Biologicals Inc. (Campbell, CA, USA). The activity of LeTx was determined by its cytolytic effect on murine macrophage cells RAW264.7. LPS was purchased from Sigma.

2.3. Activation of PBMCs with B. anthracis $C W, L F$, and $P A$. The cells were activated in 24 -well plastic plates with different concentrations of anthrax $\mathrm{CW}$ and various combinations of the LF with PA for 24, 48, and $72 \mathrm{~h}$. There was no visible cell lysis in LF, PA, and LeTx treated groups. The ratio of PA and LF used to form a LeTx in vitro is $5: 1$. Nonactivated cells were used as a negative control. The supernatants were harvested and tested for cytokine production using the ELISA assay and a flow cytometer.

2.4. Measurement of Cytokine Secretion. ELISA was used for quantitative measurement of released TNF- $\alpha$, IL- $1 \beta$, IL-2, IL6 , IL-10, and INF- $\gamma$ in the supernatants. ELISA kits were purchased from Endogen, Inc. (Woodburn, MA, USA). After treatment of the cells, the supernatants were collected and analyzed by using ELISA according to the protocols provided by the manufacturer. The $\mathrm{BD}$-Pharmingen cytometric bead array (CBA) was used to test the cytokine panel of IFN- $\gamma$, TNF- $\alpha$, IL-2, IL-4, IL-6, and IL-10 in the PBMC culture supernatants. This method uses flow cytometry to measure soluble analytes in a particle-based immunoassay as described previously [18]. Data was acquired using BD CellQuest software and analyzed using FCAP Array software provided by $\mathrm{BD}$. The lower limit of detection for all cytokines in this assay was $20 \mathrm{pg} / \mathrm{mL}$.

2.5. Statistical Analysis. All the experiments were repeated at least three times and GraphPad Prism software was used for statistical analysis. Student's $t$-test was used to compare the concentration of the tested cytokines under different conditions. A statistically significant difference was assumed at $P<0.05$.

\section{Results}

3.1. Bacterial CW but Not LeTx Induces Production of Proinflammatory Cytokines. The release of the proinflammatory 


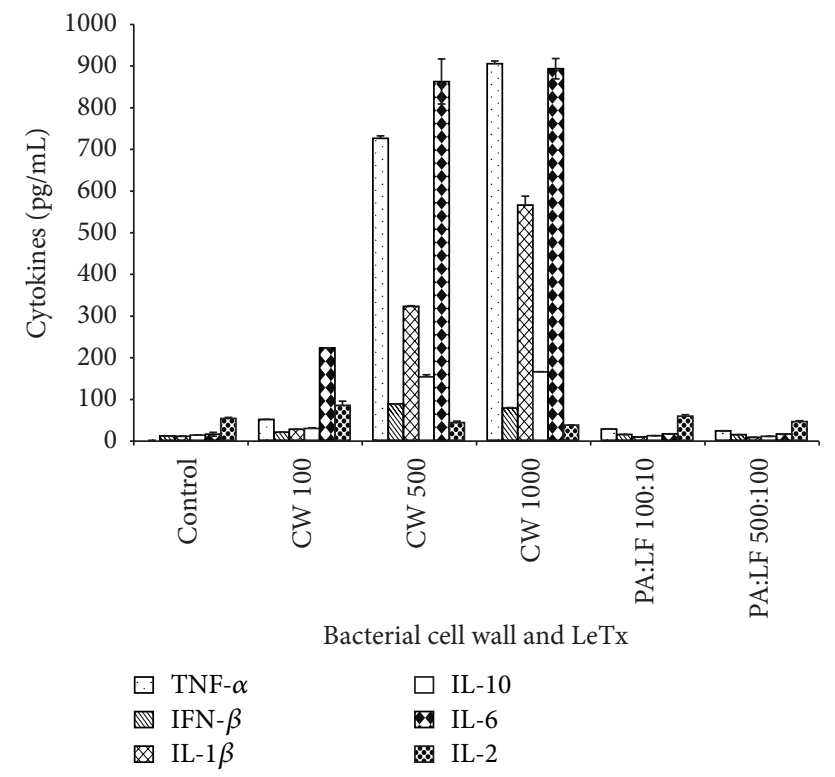

FIGURE 1: Cytokine release from human PBMCs after incubation with different amounts of $B$. anthracis cell wall preparation and LeTx. After 24 hours of incubation, the culture supernatants were collected and the cytokine concentration was measured by using ELISA. Data in the figure represents 6 donor repeats and is calculated as Mean \pm SE. Each sample was tested in triplicate.

cytokines from the human PBMCs in response to different concentrations of CW and LeTx is presented in Figure 1. PBMCs were treated with bacterial CW and LeTx at different concentrations for $24 \mathrm{~h}$ and the supernatants were harvested for testing the concentration of TNF- $\alpha$, IFN- $\gamma$, IL- $1 \beta$, IL10, IL-6, and IL-2 using ELISA assay. Among the tested groups, $1000 \mathrm{ng} / \mathrm{mL}$ of bacterial CW group induced the highest release of the cytokines compared to the control samples. To determine if the secretion of the cytokines from the PBMCs followed the CW dose dependent manner, up to $5000 \mathrm{ng} / \mathrm{mL}$ of the CW was tested. There were no significant changes in the cytokine production when the CW dose was increased from 1000 to $5000 \mathrm{ng} / \mathrm{mL}$ (data not shown). Since the production of IFN- $\gamma$ at $24 \mathrm{~h}$ was relatively low, we further extended the incubation time. An increased production of IFN- $\gamma$ was achieved with prolonged stimulation time (Figure 2). These results indicate that the expression of IFN- $\gamma$ takes a longer time than other cytokines. Meanwhile, the PBMCs were treated with different concentrations of PA (100 ng and $500 \mathrm{ng} / \mathrm{mL}$ ) and LF (20 ng and $100 \mathrm{ng} / \mathrm{mL}$ ) at a ratio of $5: 1$. It is clear that there was no production of the cytokines following exposure to various concentrations of LeTx. In contrast, there was significant production of the proinflammatory cytokines by the PBMCs induced by the CW preparation. The highest release of the cytokines was observed in the group of $1000 \mathrm{ng} / \mathrm{mL}$ of the CW. In this group, the concentrations of TNF$\alpha$, IL-6, and IL- $1 \beta$ were 900,890 , and $570 \mathrm{pg} / \mathrm{mL}$, which were significantly higher than the control and LeTx treated

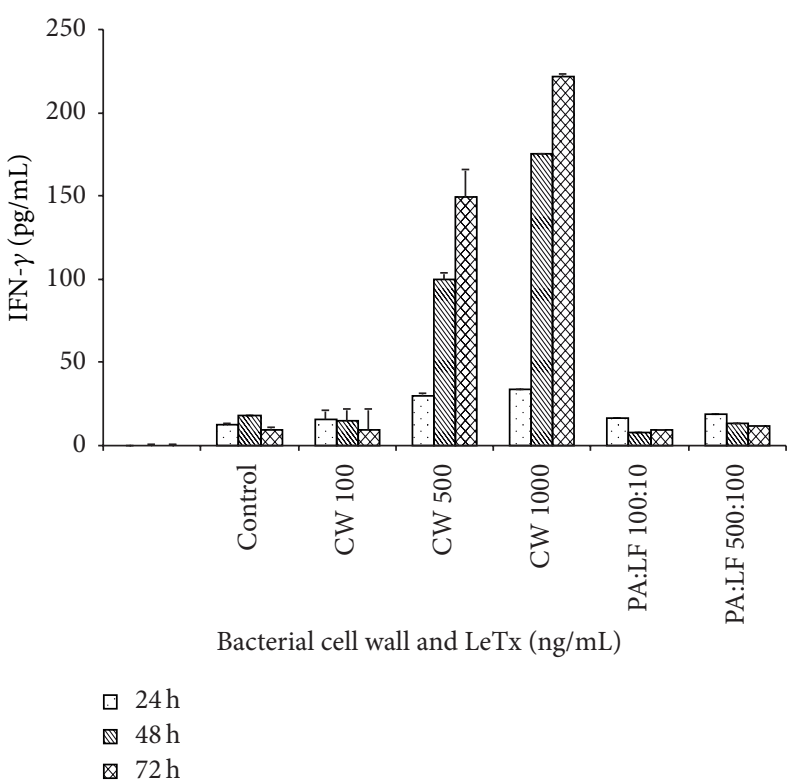

FIgure 2: Time course of IFN- $\gamma$ production from human PBMCs after incubation with different amounts of $B$. anthracis cell wall preparation and LeTx. Supernatants were harvested at 24, 48, and 72 hours, respectively, after the incubation. The amount of released IFN- $\gamma$ was measured by using ELISA. Each sample was tested in triplicate. Data in the figure represent samples from 6 different donors.

groups $(P<0.001)$. The production of IL-10 $(164 \mathrm{pg} / \mathrm{mL})$ and IFN- $\gamma(77 \mathrm{pg} / \mathrm{mL})$ after $24 \mathrm{~h}$ incubation was relatively low but still significantly higher than the control group $(P<0.001, P=0.021)$. The concentration of the tested cytokines in the control group was all below $30 \mathrm{pg} / \mathrm{mL}$. No significant IL-2 release was observed in all groups as compared to control $(P=0.42)$. No difference in the viability of the cells was observed during the activation period.

3.2. Anthrax LeTx Inhibits the Production of the Proinflammatory Cytokines. To determine if there is synergic or antagonistic effect between CW and anthrax toxin in inducing cytokine production, human PBMCs were treated with CW, anthrax toxin components (PA, LF, and $\mathrm{EF}$ ), either alone or in combination for $72 \mathrm{~h}$. Human Th1/Th2 CBA kit was used to test the concentration of IL-2, IL-4, IL-6, IL-10, TNF- $\alpha$, and IFN- $\gamma$ in the supernatants. It was obvious that the bacterial CW could efficiently induce the release of IL-6, IL-10, TNF$\alpha$, and INF- $\gamma$. However, the LeTx alone did not induce any production of the cytokines (Figure 3). Interestingly, the effects of the CW on the cytokine production were completely eliminated in the presence of the LeTx. We further tested the individual components of anthrax toxin. PA, EF, LF alone, and EdTx were all able to induce certain amount of the production. These results indicate that anthrax LeTx is only one strong inhibitor for the proinflammatory cytokine production in this study. The major causative element to block the cytokine release is LF. Figure 3 is a typical dot 

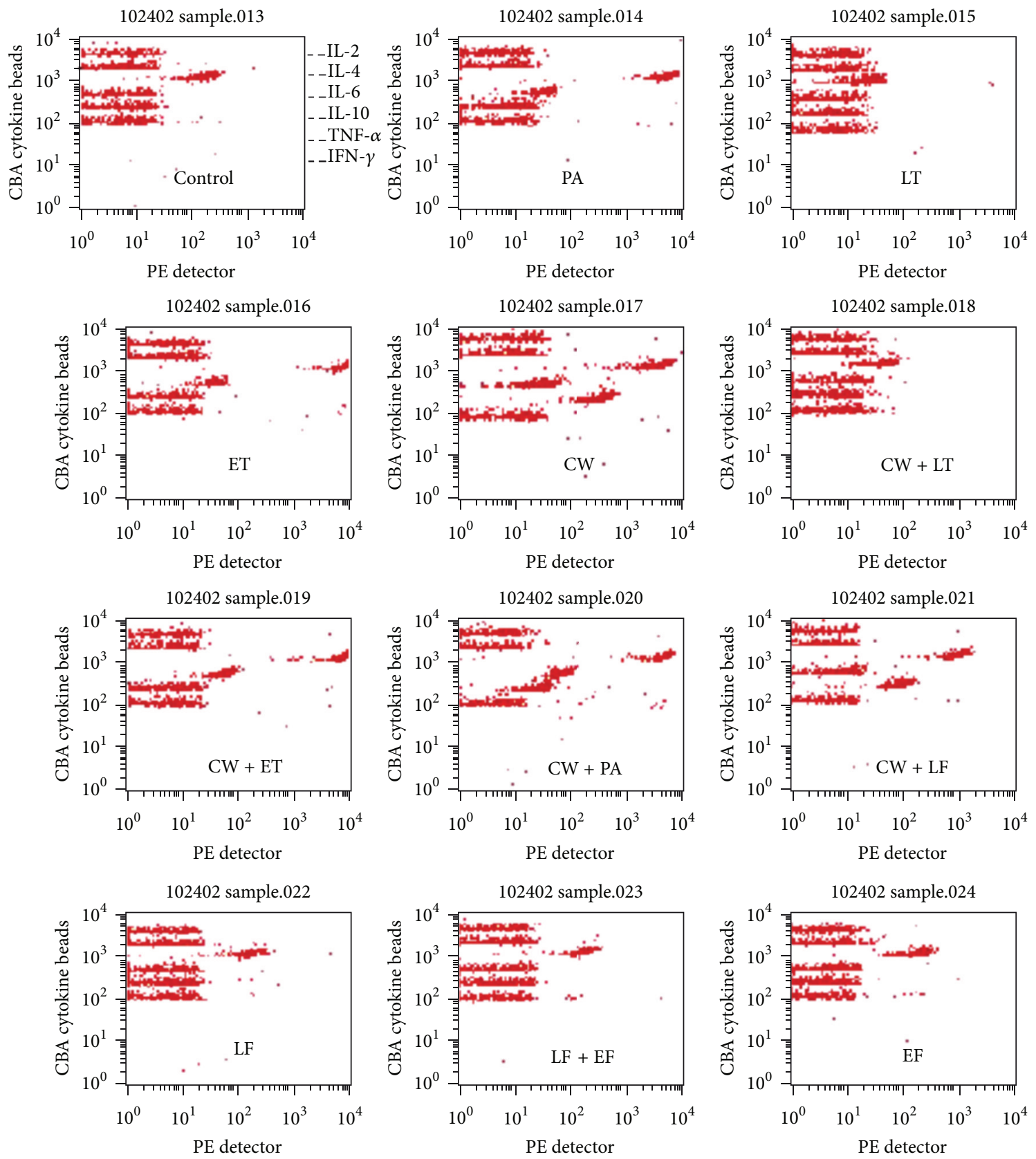

FIGURE 3: Th1/Th2 cytokine panel determination using cytometric bead array (CBA) assay. Human PBMCs were treated with B. anthracis cell wall preparation and various elements of anthrax toxin, either individually or in combination, for $72 \mathrm{~h}$. Supernatants were harvested and assayed to determine the concentration of 6 different cytokines. The beads were conjugated with antibodies against corresponding cytokines. Secondary antibody conjugated with fluorescence dye PE was used as a detector. SSC versus FSC were used to locate the position of cytokine beads. The dots from top to bottom represent IL-2, IL-4, IL-6, IL-10, TNF- $\alpha$, and IFN- $\gamma$, respectively. FL3 versus FL2 were used to determine the concentration of cytokines. With increased concentration, the dot plots move to right side. The intensity of the fluorescence is correlated with the concentration of tested cytokines. CW: cell wall, LT: lethal toxin, ET: edema toxin, LF: lethal factor, EF: edema factor, and PA: protective antigen.

plot of cytokine release from a healthy young male donor as measured by using CBA. The similar cytokine release pattern was observed from all 6 health donors. Figure 4 is the quantitative results of the cytokine production $(n=$ 6) as determined by using CBA. There was no significant production of IL-2 and IL-4 in any case. However, notable amounts of IL- 6 and IL-10 were detected as compared to negative controls $(P<0.001)$. TNF- $\alpha$ was only observed in $\mathrm{CW}$ activation group. IFN $-\gamma$ release was induced by $\mathrm{CW}$ and PA with a smaller amount compared to IL-6 and IL-10. 


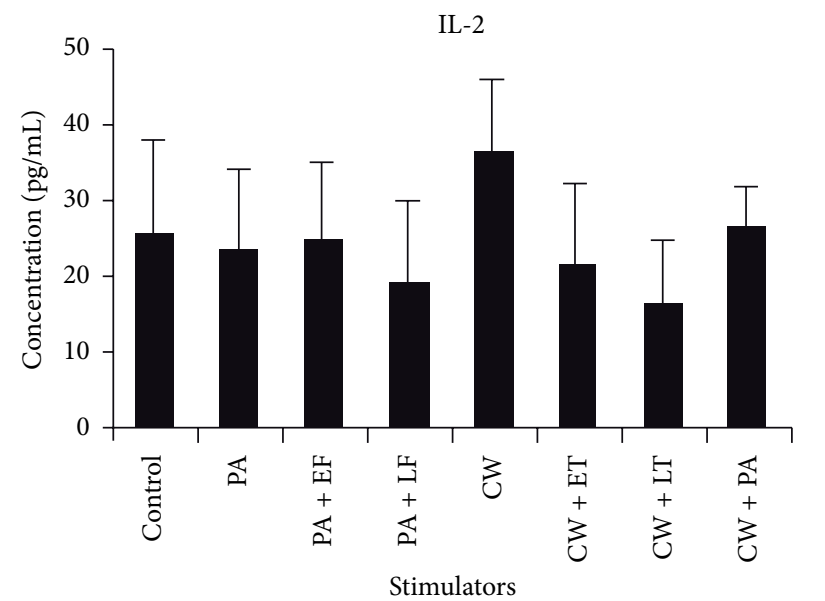

(a)

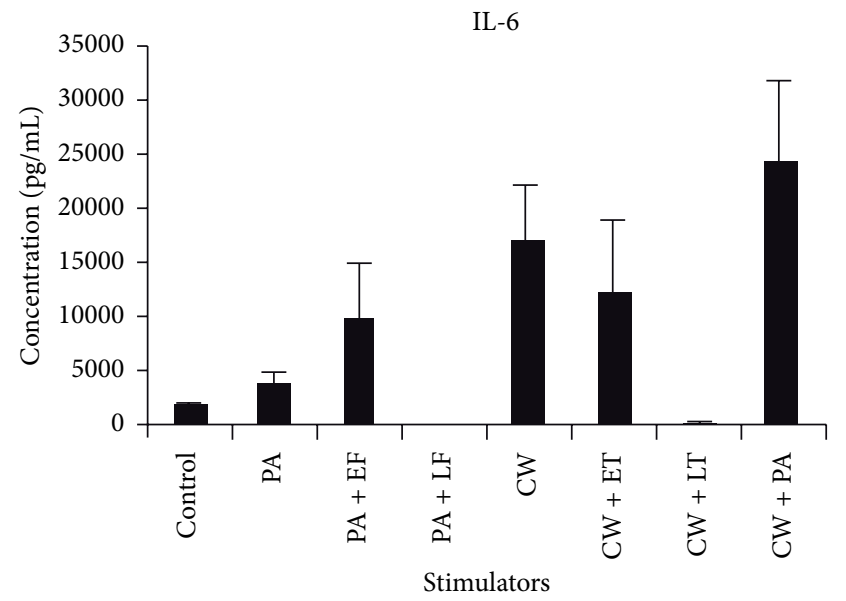

(c)

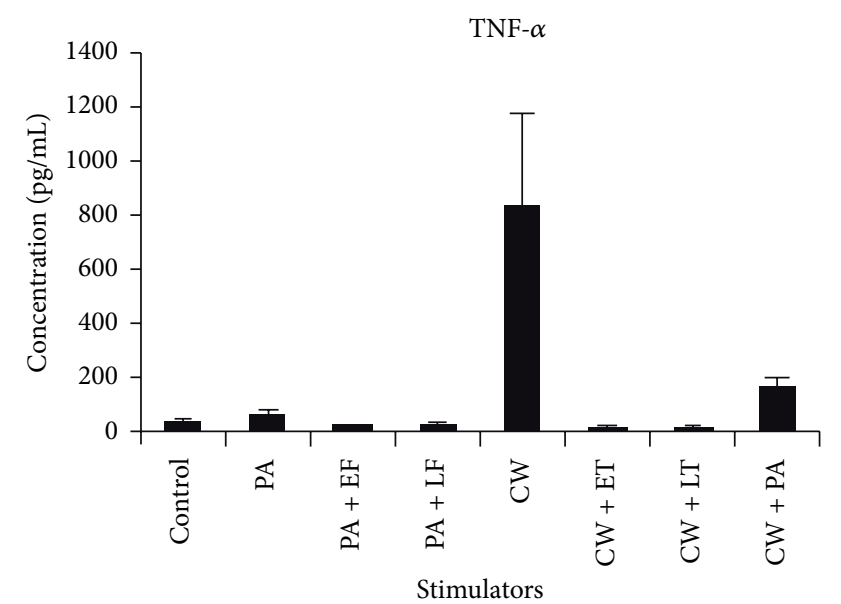

(e)

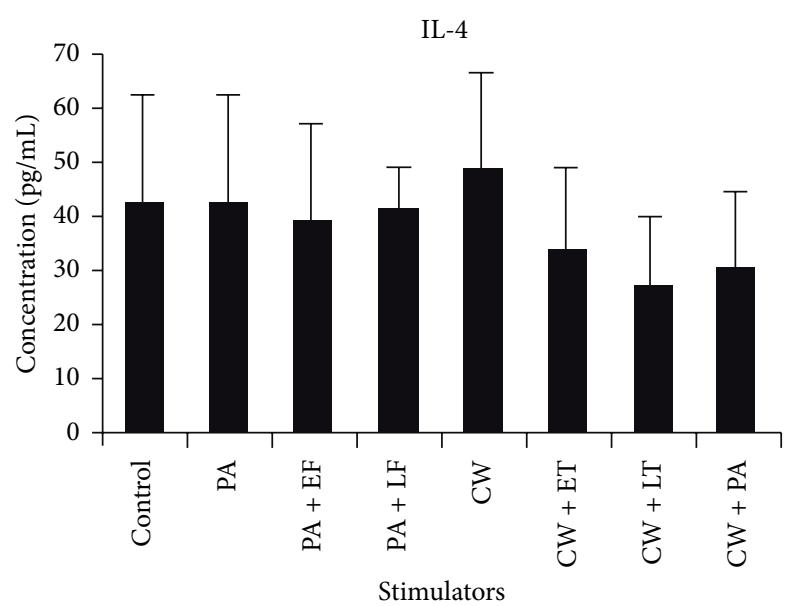

(b)

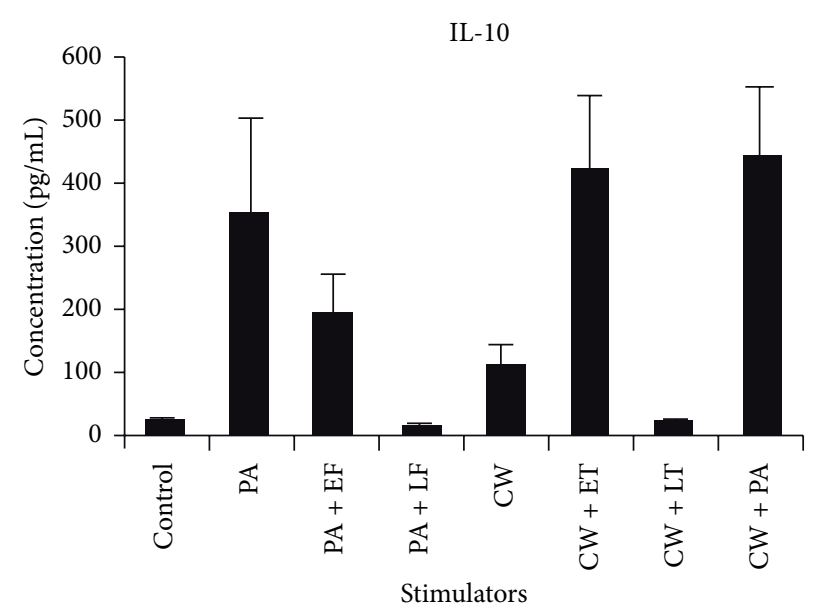

(d)

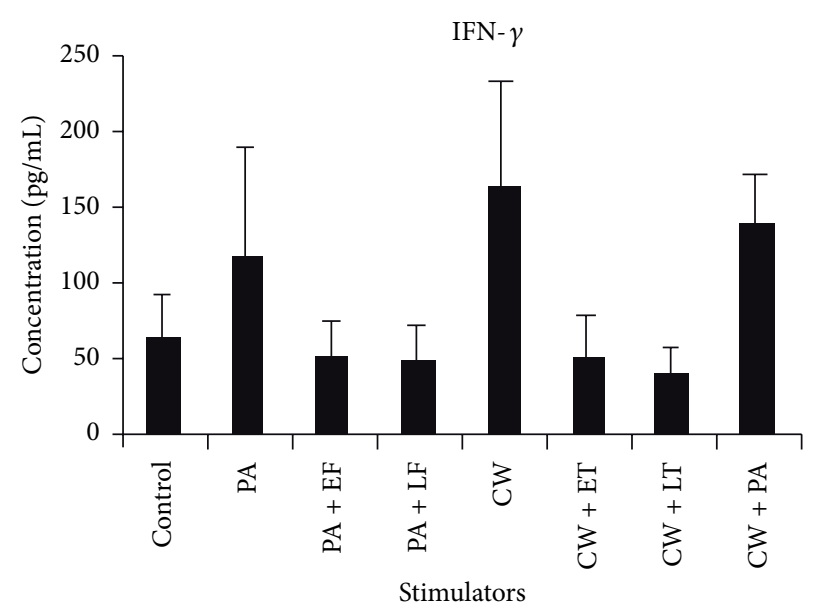

(f)

FIgURE 4: Quantitative analysis of Th1/Th2 cytokines produced by human PBMCs using BD-Pharmingen CBA kit. Human PBMCs were treated with $B$. anthracis $\mathrm{CW}$ and anthrax toxin components, either individually or in combination, for $72 \mathrm{~h}$ at $37^{\circ} \mathrm{C}$. Supernatants were harvested, stained with antibody-conjugated beads, and analyzed with FACS Calibur. The concentration of each cytokine was calculated with BD CBA software. Data were derived from 6 different donors. (a): IL-2, (b): IL-4, (c): IL-6, (d): IL-10, (e): TNF- $\alpha$, and (f): IFN- $\gamma$. 


\section{Discussion}

It was believed that low levels of anthrax LeTx can induce the release of cytokines such as TNF- $\alpha$ and IL- $1 \beta[1,5,10]$. It was also presumed that LF only affects macrophages, causing a mass production of the proinflammatory cytokines, which in turn induced shock and death in the late stage of the infection. High concentration of LeTx resulted in cytolysis of macrophage [18]. This classical presumption of the pathogenesis of anthrax infection has been heavily challenged by recent studies. Several independent groups have reported that macrophages do not express the proinflammatory cytokines after exposure to anthrax LeTx [11, 12]. In this study, we presented the first evidence that anthrax LeTx inhibits the cytokine production by the human PBMCs.

The strong immune response of PBMCs to the released proinflammatory mediators such as TNF- $\alpha$, IL- $1 \beta$, and IL6 upon stimulation by the bacterial CW suggests potential mechanisms that underlie anthrax sepsis and shock. It has been hypothesized that detrimental effects observed in anthrax infections are primarily the result of a surge of TNF- $\alpha$ and IL- $1 \beta$ released by the activated macrophages. High levels of IL- $1 \beta$ and TNF- $\alpha$ are known to be major shock mediators and could contribute to the "dramatic death" seen in the latestage anthrax infection. The fact that $B$. anthracis $\mathrm{CW}$ can activate the PBMCs to release the proinflammatory mediators indicates that the bacterial CW would play an important role in the pathogenesis of anthrax infection.

A homeostatic status of the cytokine network is critical in many different physical processes including cell growth and differentiation, development, and tissue repairing. One of the main functions of cytokines is to mediate interaction between the immune and inflammatory systems. Depending on the stage of inflammation or biological effect determined, the same cytokine might be anti-inflammatory [6]. During initial stages of infection, these inflammatory mediators at a low concentration (cytokines, chemokines, and small peptides) are essential as they enable the host to identify, target and finally eliminate pathogens. During the process of antigen presentation, the antigen processing cells (APC) secrete several mediators to stimulate $\mathrm{T}$ - and B-lymphocytes in order to develop specific immunity against the pathogen. $\mathrm{CD}^{+} \mathrm{T}$ lymphocytes exert their function primarily through secreted cytokines. Three distinguishable subpopulations of $\mathrm{CD}^{+}$cell have been defined. Th1 cells secrete IL-2, INF $\gamma$, TNF- $\alpha$, and IL-12p35-40. Th2 cells produce IL-4, IL-5, IL-10, and IL-13, and Th3 cells express CD4/CD25 and release tumor necrosis factor- $\beta[19,20]$. Generally, Th1 cells stimulate cellular mediated immunity and IgG2a production whereas Th2 cells favor the humoral immunity. This indicates that humoral antibodies against $B$. anthracis play an important role in controlling the spread of the pathogens. The cytokine panel used in this study revealed an initial polarization of the immune response to anthrax infection.

We have reported that LeTx can induce apoptosis in both murine macrophage and human PBMCs [21, 22]. The phagocytic function of macrophage is suppressed by LeTx.
In this study, we further show that LeTx inhibits the proinflammatory cytokine production induced by CW components. Several groups have reported that LeTx reduces the production of a number of inflammatory mediators including transcription factors, chemokines, and cytokines [23, 24]. The mechanism of the inhibition is not due to degradation of the mediators after release from the cells but rather suppression of expression of the mediators $[25,26]$. Thus, we presume that immune response to the invading pathogen, particularly the function of macrophages, was overwhelmingly suppressed by LeTx during the process of the infection. This enables the $B$. anthracis organism to bypass the innate immunity by interrupting the defense mechanisms adapted in the early stage of infection. Thus, approaches to improve the function of macrophages should be considered for prophylaxis and early-stage treatment purposes in the future studies.

In summary, the following conclusions are derived from this study. First, CW rather than LeTx induces the production of the proinflammatory cytokines in anthrax infection. These results further confirm previous reports [11, 12]. Secondly, the unique and novel information we provide here is that LeTx inhibits the production of proinflammatory cytokine induced by CW components. The ability of LeTx to suppress cell wall induced cytokines has shed new light on functional properties of $B$. anthracis components.

\section{Acknowledgments}

This work was supported by contracts between the Defense Advanced Research Projects Agency (DARPA) and AFG Biosolutions, Inc. The authors want to thank Dr. Joannna Kubler-Kielb at NIDDK, NIH, for providing the CW preparation. They also want to thank Ms. Jennifer Cardwell for doing part of the experiments for this study.

\section{References}

[1] T. C. Dixon, M. Meselson, J. Guillemin, and P. C. Hanna, "Anthrax," The New England Journal of Medicine, vol. 341, no. 11, pp. 815-826, 1999.

[2] A. M. Friedlander, "The anthrax toxins," in Trafficking of Bacterial Toxins, C. B. Saelingir, Ed., pp. 121-138, CRC Press, Boca Raton, Fla, USA, 1990.

[3] S. H. Leppla, "Anthrax toxin edema factor: a bacterial adenylate cyclase that increases cyclic AMP concentrations in eukaryotic cells," Proceedings of the National Academy of Sciences of the United States of America, vol. 79, no. 10 I, pp. 3162-3166, 1982.

[4] S. H. Leppla, "The anthrax toxin complex," in Sourcebook of Bacterial Protein Toxins, J. Alouf, Ed., pp. 277-302, Academic Press, New York, NY, USA, 1991.

[5] P. Hanna, "Lethal toxin actions and their consequences," Journal of Applied Microbiology, vol. 87, no. 2, pp. 285-287, 1999.

[6] P. C. Hanna, B. A. Kruskal, R. A. Ezekowitz, B. R. Bloom, and R. J. Collier, "Role of macrophage oxidative burst in the action of anthrax lethal toxin," Molecular Medicine, vol. 1, no. 1, pp. 7-18, 1994.

[7] N. S. Duesbery and G. F. V. Woude, "Anthrax toxins," Cellular and Molecular Life Sciences, vol. 55, no. 12, pp. 1599-1609, 1999. 
[8] J. O'Brien, A. Friedlander, and T. Dreier, "Effects of anthrax toxin components on human neutrophils," Infection and Immunity, vol. 47, no. 1, pp. 306-310, 1985.

[9] B. E. Ivins, S. L. Welkos, S. F. Little, M. H. Crumrine, and G. O. Nelson, "Immunization against anthrax with Bacillus anthracis protective antigen combined with adjuvants," Infection and Immunity, vol. 60, no. 2, pp. 662-668, 1991.

[10] P. C. Hanna, D. Acosta, and R. J. Collier, "On the role of macrophages in anthrax," Proceedings of the National Academy of Sciences of the United States of America, vol. 90, no. 21, pp. 1019810201, 1993.

[11] J. L. Erwin, L. M. Dasilva, S. Bavari, S. F. Little, A. M. Friedlander, and T. C. Chanh, "Macrophage-derived cell lines do not express proinflammatory cytokines after exposure to Bacillus anthracis lethal toxin," Infection and Immunity, vol. 69, no. 2, pp. 1175-1177, 2001.

[12] R. Pellizzari, C. Guidi-Rontani, G. Vitale, M. Mock, and C. Montecucco, "Anthrax lethal factor cleaves MKK3 in macrophages and inhibits the LPS/IFN $\gamma$-induced release of NO and TNF $\alpha$, FEBS Letters, vol. 462, no. 1-2, pp. 199-204, 1999.

[13] C. R. H. Raetz, "Biochemistry of endotoxins," Annual Review of Biochemistry, vol. 59, pp. 129-170, 1990.

[14] B. Henderson and M. Wilson, "Cytokine induction by bacteria: beyond lipopolysaccharide," Cytokine, vol. 8, no. 4, pp. 269-282, 1996.

[15] B. Henderson and M. Wilson, "Modulins: a new class of cytokine-inducing, pro-inflammatory bacterial virulence factor," Inflammation Research, vol. 44, no. 5, pp. 187-197, 1995.

[16] B. Henderson, S. Poole, and M. Wilson, "Bacterial modulins: a novel class of virulence factors which cause host tissue pathology by inducing cytokine synthesis," Microbiological Reviews, vol. 60, no. 2, pp. 316-341, 1996.

[17] A. E. Medvedev, T. Flo, R. R. Ingalls et al., "Involvement of CD14 and complement receptors CR3 and CR4 in nuclear factor- $\kappa \mathrm{B}$ activation and TNF production induced by lipopolysaccharide and group B streptococcal cell walls," Journal of Immunology, vol. 160 , no. 9, pp. 4535-4542, 1998 .

[18] R. Chen, R. Lowe, J. D. Wilson et al., "Simultaneous quantification of six human cytokines in a single sample using microparticle-based flow cytometric technology," Clinical Chemistry, vol. 45, pp. 1693-1694, 1999.

[19] M. Moser and K. M. Murphy, "Dendritic cell regulation of TH1TH2 development," Nature Immunology, vol. 1, no. 3, pp. 199205, 2000.

[20] M. F. Neurath, S. Finotto, and L. H. Glimcher, "The role of $\mathrm{TH} 1 / \mathrm{TH} 2$ polarization in mucosal immunity," Nature Medicine, vol. 8, no. 6, pp. 567-573, 2002.

[21] S. G. Popov, R. Villasmil, J. Bernardi et al., "Lethal toxin of Bacillus anthracis causes apoptosis of macrophages," Biochemical and Biophysical Research Communications, vol. 293, no. 1, pp. 349355, 2002.

[22] S. G. Popov, R. Villasmil, J. Bernardi et al., "Effect of Bacillus anthracis lethal toxin on human peripheral blood mononuclear cells," FEBS Letters, vol. 527, no. 1-3, pp. 211-215, 2002.

[23] G. Whiting, M. Baker, and S. Rijpkema, "Development of an in vitro potency assay for anti-anthrax lethal toxin neutralizing antibodies," Toxins, vol. 4, no. 1, pp. 28-41, 2012.

[24] A. Cleret-Buhot, J. Mathieu, J. N. Tournier, and A. QuesnelHellmann, "Both lethal and edema toxins of Bacillus anthracis disrupt the human dendritic cell chemokine network," PLoS One, vol. 7, no. 8, Article ID e43266, 2012.
[25] C. M. Gonzales, C. B. Williams, V. E. Calderon et al., "Antibacterial role for natural killer cells in host defense to Bacillus anthracis," Infection and Immunity, vol. 80, no. 1, pp. 234-242, 2012.

[26] E. K. Dumas, P. M. Cox, C. O. Fullenwider et al., "Anthrax lethal toxin-induced gene expression changes in mouse lung," Toxins, vol. 3, no. 9, pp. 1111-1130, 2011. 

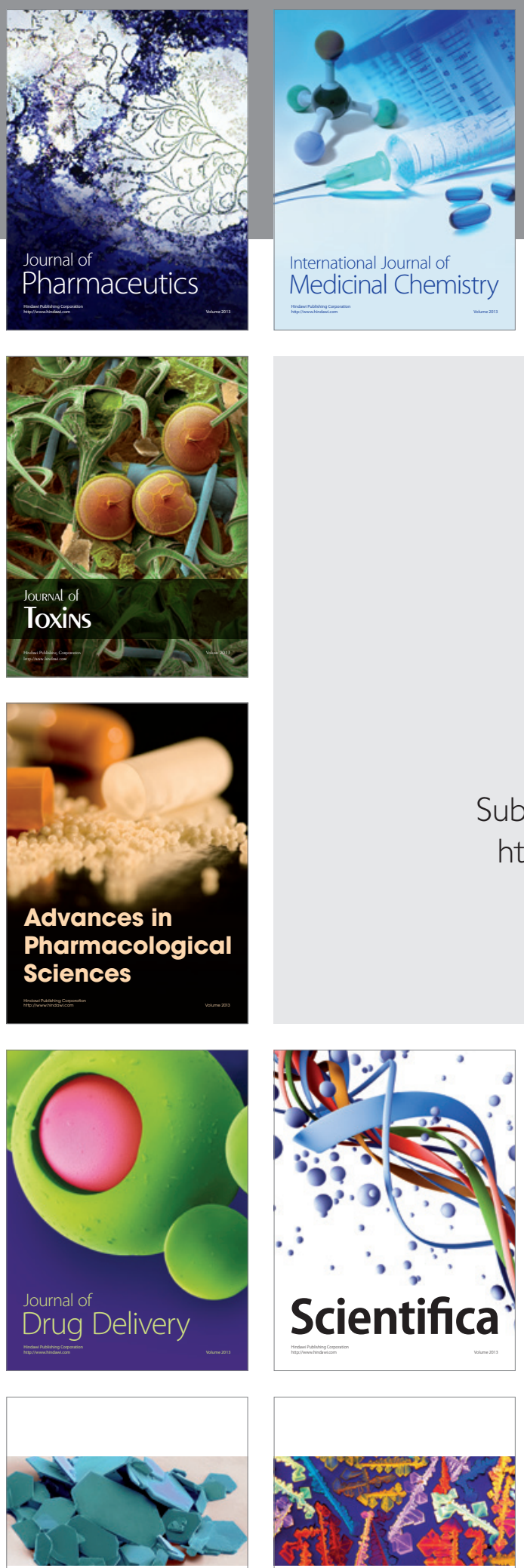

ISRN

Pharmacology

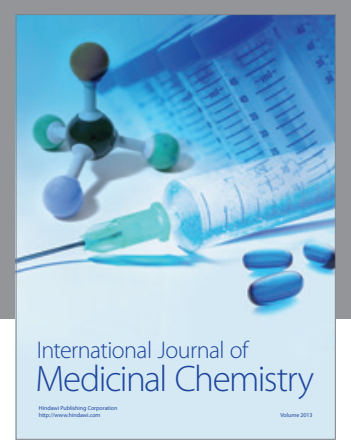

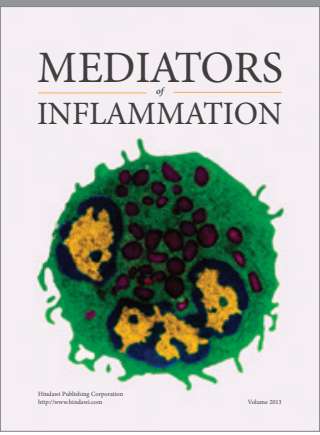
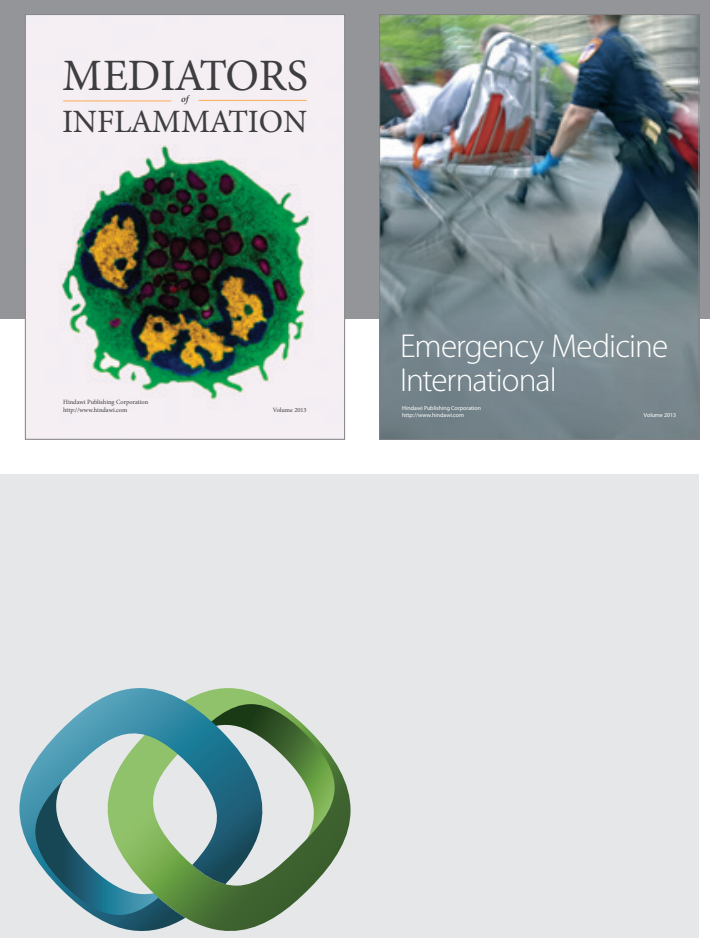

\section{Hindawi}

Submit your manuscripts at http://www.hindawi.com

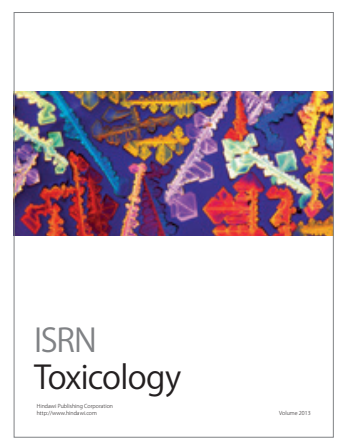

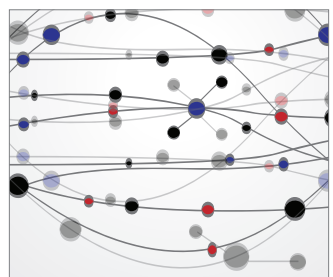

The Scientific World Journal
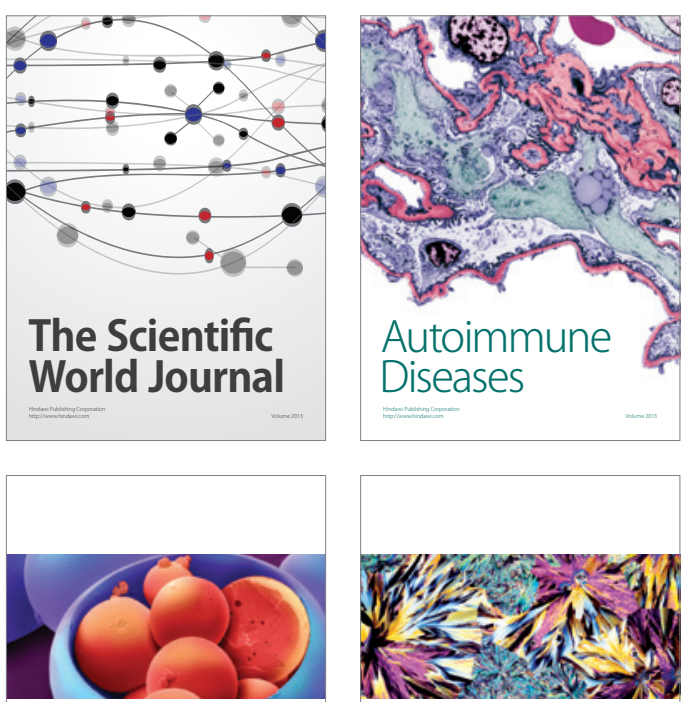

ISRN

Pharmaceutics

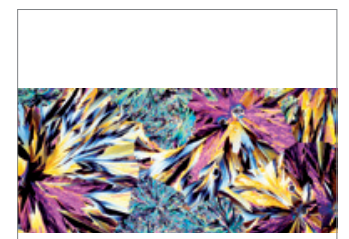

ISRN

Pain
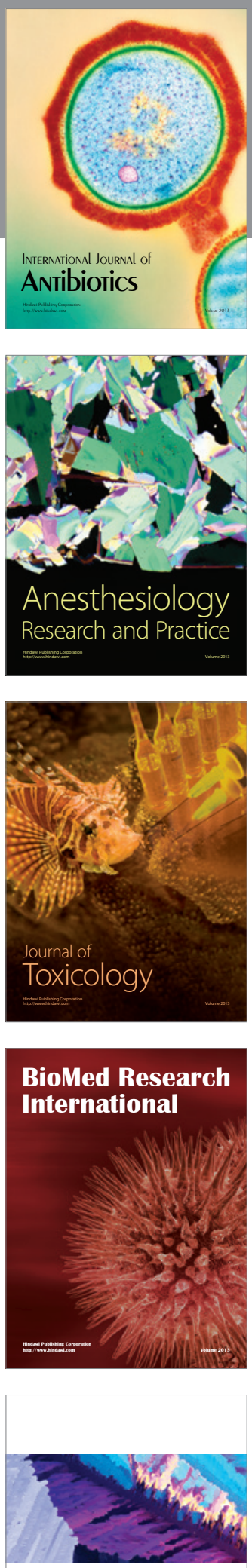

ISRN

Medicinal

Chemistry 